\title{
Levantamento de aditivos alimentares em produtos alimentícios voltados para o público infantil
}

\author{
Luiza Vargas Mascarenhas Braga ${ }^{1}$, Alessandro Rangel Carolino Sales Silva ${ }^{2}$ e Lucilene \\ Rezende Anastácio ${ }^{3}$
}

O consumo de alimentos ultraprocessados pode ser especialmente prejudicial na infância, uma vez que as crianças são consideradas mais vulneráveis aos efeitos dos aditivos alimentares. Objetivou-se avaliar, de acordo com a informação presente nos rótulos, os tipos de aditivos alimentares presentes em alimentos destinados ao público infantil. Trata-se de estudo transversal, descritivo, no qual se compilou os tipos de aditivos presentes na lista de ingredientes de oito categorias (bebidas com sabor de frutas, bebidas lácteas, biscoitos recheados, bolos, cereais matinais, gelatinas, salgadinhos de milho e iogurtes) de produtos com apelo infantil $(n=409)$ coletados em supermercados de Belo Horizonte-MG, durante segundo semestre de 2018. Apenas 19 (4,6\%) produtos não possuíam algum tipo de aditivo. As categorias dos bolos $(8,3 \pm 2,1)$ e das gelatinas $(8,3 \pm 1,2)$ apresentaram maior média de aditivos e a categoria dos cereais matinais, menor $(2,2 \pm 1,6)$. Os aditivos alimentares mais encontrados foram: aromatizantes $(79 \% ; n=323)$, corantes $(56 \% ; n=229)$ e emulsificantes $(36 \% ; n=148)$. Conclui-se que os alimentos voltados ao público infantil possuem, em sua maioria, pelo menos um tipo de aditivo alimentar. Foram encontradas majoritariamente as classes dos aromatizantes, corantes e emulsificantes nos produtos avaliados.

Palavras-chave: Aditivos alimentares; Alimentos infantis; Rotulagem de alimentos; Crianças.

\section{Food additives in ultra-processed products intended for children and its impacts on health}

Consumption of ultra-processed foods can be especially harmful in childhood, as children are considered more vulnerable to the effects of food additives, which have had an acceptable daily intake (ADI) established for adults. The objective was to evaluate, according to the information on the labels, the types of food additives present in foods targeted at children. It is a cross-sectional, descriptive study which compiled the types of additives in the ingredient list of eight categories (fruit drink, dairy drinks, sandwich cookies, cakes, breakfast cereals, jellies, corn chips, and yogurts) of products with child appeal $(\mathrm{n}=409)$ collected in supermarkets in Belo Horizonte-MG, in the second half of 2018. Only $19(4.6 \%)$ products had no additives. The cakes $(8,3 \pm 2.1)$ and gelatins $(8,3 \pm 1.2)$ categories presented the highest average of additives and the morning cereals category, the lowest $(2,0 \pm 1.6)$. The most commonly found food additives were: flavoring $(79 \% ; n=323)$, colorings $(56 \% ; n=229)$ and emulsifiers $(36 \% ; n=148)$. It was mainly found the classes of flavorings, colorings and emulsifiers.

\footnotetext{
${ }^{1}$ Universidade Federal de Minas Gerais. E-mail: luizavmb@gmail.com; ID ORCID: https://orcid.org/0000-0002-6822-7688

2 Universidade Federal de Minas Gerais. E-mail: alessandrorcss@hotmail.com; ID ORCID: https://orcid.org/0000-0003-0291-3578

3 Universidade Federal de Minas Gerais. E-mail: lucilene.rezende@gmail.com; ID ORCID: https://orcid.org/0000-0002-2269-0722. Endereço para correspondência: Departamento de Alimentos, Faculdade de Farmácia, Av. Pres. Antônio Carlos, 6627 - Pampulha, Belo Horizonte - MG, 31270-901
} 
Keywords: Food additives; Infant food; Food labeling; Child.

Submetido em: 10/06/2020

Aceito em: 11/12/2020

\section{INTRODUÇÃO}

Com as inovações tecnológicas industriais, os aditivos alimentares passaram a fazer parte das formulações dos produtos alimentícios, a fim de promover maior estabilidade e palatabilidade ${ }^{[1]}$. De acordo como o Codex Alimentarius, um documento referência para estabelecer padrão de qualidade e inocuidade dos produtos, aditivo é qualquer substância que não se consome normalmente como alimento, nem tão pouco se usa como ingrediente básico, tendo ou não valor nutritivo, e a adição têm como intenção exercer alguma função tecnológical 2,3$]$. Com o intuito de garantir que os aditivos alimentares não ocasionem danos à saúde do consumidor, o documento regulatório supracitado estipula a Ingestão Diária Admissível (IDA), quantidade ingerida diariamente sem risco a saúde, expressa em relação ao peso corporal[3].

A publicação mais recente do Guia Alimentar da População Brasileira categoriza os alimentos de acordo com o grau de processamento que são submetidos ${ }^{[4]}$. Os alimentos in natura $\mathrm{e}$ minimamente processados são aqueles que não são submetidos a nenhum processamento ou apenas algum processo físico que não altera a composição do alimento[ ${ }^{[4]}$. Os alimentos processados são alimentos in natura adicionados de açúcares, sal e óleo (sendo esses três classificados como ingredientes), e os ultraprocessados são formulações industriais compostos minimamente por alimentos in natura e, majoritariamente, por substâncias industriais, como os aditivos ${ }^{[4]}$. As recomendações do Guia sugerem que o último grupo tenha o consumo evitado[4].

Com a exposição de crianças a grande quantidade de alimentos ultraprocessados, associadas a poucas práticas de Educação Alimentar e Nutricional promovida pelas Instituições e a complexidade de interpretação da rotulagem de alimentos, essa população torna-se mais frágil[5,6]. Além disso, essa faixa etária não está fisiologicamente preparada para metabolizar e excretar muitas das substâncias químicas que são ingeridas e não é capaz de controlar o consumo de alimentos ultraprocessados, sendo constantemente instigados pelo excesso de publicidade ${ }^{[7]}$.

Dessa forma, o presente estudo objetivou analisar, listar e descrever os tipos de aditivos alimentares presentes em alimentos destinados ao público infantil.

\section{MÉTODOS}

Estudo transversal descritivo, no qual foram compilados os tipos e quantidades de aditivos presentes nos produtos de oito categorias coletadas voltadas para o público infantil (bebidas com sabor de frutas, bebidas lácteas, biscoitos recheados, bolos, cereais matinais, gelatinas, salgadinhos de milho e iogurtes). Estas foram selecionadas dentro do grupo de alimentos mais populares com apelo infantil comercializados em mercado brasileiro e de acordo com estudo de Ares e colaboradores ${ }^{[8,9]}$.

Foi realizado levantamento online dos produtos das oito categorias, seguindo critérios estabelecidos também no estudo de Ares e colaboradores $^{[9]}$ (i. presença de personagens animados, personagens televisivos ou referência a filmes; ii. presença de frases que sugerem o consumo do produto em questão por crianças; iii. existência de design infantilizado do produto por meio de cores e ilustrações) ${ }^{[5]}$, alcançando à listagem de 532 produtos. Procedeu-se com a coleta de dados, no segundo semestre de 2018, em supermercados de Belo Horizonte-MG e região metropolitana. Produtos não encontrados foram excluídos $(\mathrm{n}=123)$, chegando-se à listagem final de 409 produtos.

Os aditivos foram agrupados de acordo com a classe que antecede o nome do aditivo, dispostas na lista de ingredientes, conforme previsto nas 
legislações que regem Padrão de Identidade e Qualidade.

Utilizou-se o software de armazenamento de dados Epicollect5, no qual elaborou-se questionário de coleta com as informações pertinentes ao estudo. $\mathrm{E}$, por meio da lista de ingredientes registrada, foram listados os aditivos de cada produto, sendo separados por classes. Os dados foram compilados no programa Microsoft Excel 2010, sendo identificados os principais aditivos dos produtos da amostra. Além disso, realizou-se análise de cluster dos produtos avaliados, na melhor combinação possível, os quais foram agrupados considerando-se a prevalência das 13 classes de aditivos alimentares mais presentes nas oito categorias de produtos avaliados, com auxílio do software Statistical Package for Social Sciences versão 17.0.

\section{RESULTADOS}

Foram avaliados 409 rótulos, sendo majoritária a categoria dos biscoitos recheados $(\mathrm{n}=103)$. A média geral foi de 3,8 aditivos por produto analisado. A categoria dos bolos $(8,3 \pm 2,1) \mathrm{e}$ das gelatinas $(8,3 \pm 1,2)$ apresentaram maior número médio de aditivos e a categoria dos cereais matinais, menor número médio $(2,2 \pm 1,6)$ (Gráfico 1). $\mathrm{Na}$ amostra estudada, foram encontrados 17 das 23 classes de aditivos com utilização respaldada pelo Codex. O número de produtos que não apresentavam aditivos foi de apenas 19 (4,6\%), sendo estes pertencentes às categorias de salgadinhos $(4 \% ; n=$ $2)$, bebidas lácteas $(3 \% ; \mathrm{n}=1)$, bebidas à base de frutas $(15 \% ; n=10)$ e cerais matinais $(20 \% ; n=6)$.
Gráfico 1. Número de produtos alimentícios voltados ao público infantil coletados por categorias, Belo Horizonte-MG 2020.

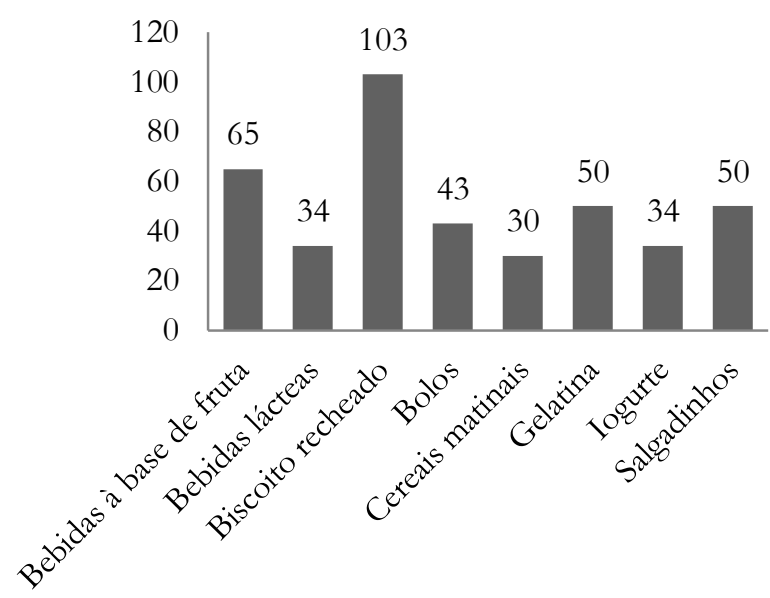

Dentre os tipos de aditivos encontrados, os aromatizantes $(88 \% ; n=360)$ (Gráfico 2) foram os mais presentes, principalmente na categoria dos biscoitos recheados, o qual foi detectado em 90 dos 103 produtos (87\%). Acredita-se que seu uso esteja relacionado com melhoria sensorial dos produtos, como citado por Polônio e colaboradores ${ }^{[10]}$. Não foi possível descobrir quais eram os aromatizantes empregados, uma vez que a RDC № 2 de 2007 permite que eles sejam declarados de forma agrupada, dificultando a classificação destes em naturais e sintéticos[11].

Gráfico 2. Número de aditivos separados por classes, encontrados em todas as categorias de produtos alimentícios destinados ao público infantil pesquisados, Belo Horizonte-MG 2018.

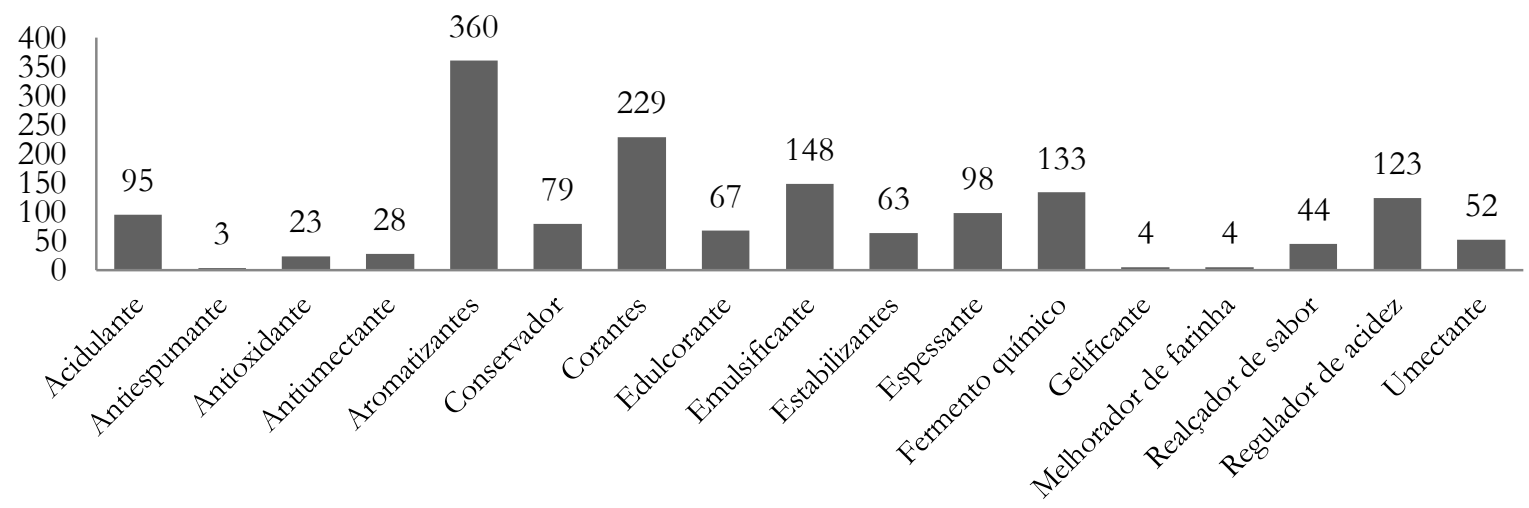


A segunda classe de aditivos mais listada foi a classe dos corantes (a qual engloba os corantes artificiais, os idênticos ao natural e os naturais) (56\%; $\mathrm{n}=229)$, presente em todos os produtos da categoria das gelatinas $(100 \% ; \mathrm{n}=50)$.
Os aromatizantes estavam entre os três primeiros aditivos mais utilizados em todas as categorias analisadas, seguidos pelos corantes, presente entre os principais em três das oito categorias analisadas, sendo elas gelatina, salgadinhos de milho e cereais matinais (Tabela 1).

Tabela 1. Classes predominantes de aditivos presentes segundo as categorias dos produtos alimentícios destinados ao público infantil pesquisados, Belo Horizonte-MG 2018.

\begin{tabular}{|c|c|c|c|}
\hline CATEGORIAS DE ALIMENTOS & CLASSES DE ADITIVOS & $\mathrm{n}$ & $\%$ \\
\hline \multirow{3}{*}{$\begin{array}{l}\text { Biscoito recheado } \\
(n=103)\end{array}$} & Emulsificante & 93 & 90 \\
\hline & Fermento químico & 91 & 88 \\
\hline & Aromatizante & 90 & 87 \\
\hline \multirow{3}{*}{$\begin{array}{l}\text { Bebidas a base de frutas } \\
(n=65)\end{array}$} & Regulador de acidez & 53 & 82 \\
\hline & Espessante & 45 & 69 \\
\hline & Aromatizante & 37 & 57 \\
\hline \multirow{3}{*}{$\begin{array}{l}\text { Gelatina } \\
(n=50)\end{array}$} & Regulador de acidez & 50 & 100 \\
\hline & Corante Artificial & 50 & 100 \\
\hline & Aromatizante & 50 & 100 \\
\hline \multirow{3}{*}{$\begin{array}{l}\text { Salgadinhos } \\
(n=50)\end{array}$} & Aromatizante & 47 & 94 \\
\hline & Realçadores de sabor & 44 & 88 \\
\hline & Corante Natural & 38 & 76 \\
\hline \multirow{3}{*}{$\begin{array}{l}\text { Bolos } \\
(n=43)\end{array}$} & Aromatizante & 42 & 98 \\
\hline & Conservantes & 39 & 91 \\
\hline & Emulsificante & 37 & 86 \\
\hline \multirow{3}{*}{$\begin{array}{l}\text { Bebidas lácteas } \\
(\mathrm{n}=34)\end{array}$} & Estabilizante & 34 & 100 \\
\hline & Aromatizante & 26 & 76 \\
\hline & Espessante & 15 & 44 \\
\hline \multirow{3}{*}{$\begin{array}{l}\text { Iogurtes } \\
(n=34)\end{array}$} & Acidulante & 34 & 100 \\
\hline & Conservante & 34 & 100 \\
\hline & Aromatizante & 33 & 97 \\
\hline \multirow{3}{*}{$\begin{array}{l}\text { Cereais matinais } \\
(\mathrm{n}=30)\end{array}$} & Aromatizante & 19 & 63 \\
\hline & Antioxidante & 10 & 33 \\
\hline & Corante Artificial & 6 & 20 \\
\hline
\end{tabular}

Os emulsificantes $(36 \% ; n=148)$ também estão em grande maioria dos produtos, estando presentes em $90 \%$ dos produtos da categoria dos biscoitos recheados. Os antioxidantes foram encontrados na categoria das bebidas à base de frutas $(20 \% ; n=13)$ e nos cereais matinais $(33 \% ; n=10)$. Já o realçador de sabor presente, glutamato monossódico, presente em todos os produtos da 
categoria dos salgadinhos de milho $(88 \% ; n=44)$ (Tabela 2).

Quanto à presença de edulcorantes $(16,4 \%$; $\mathrm{n}=67)$, estes foram encontrados na maior parte das gelatinas $(94 \% ; \mathrm{n}=47)$, em algumas bebidas à base de frutas $(26 \% ; n=17)$ e iogurtes $(9 \% ; n=3)$. A categoria das gelatinas apresentou combinação dessa classe, composta por acessulfame de potássio e mais um edulcorante, em todos os produtos que continham edulcorantes. Além disso, todos os produtos que continham ciclamato de sódio $(86 \% ; \mathrm{n}=43)$, sacarina sódica $(64 \% ; \mathrm{n}=32)$ e aspartame $(58 \% ; \mathrm{n}=29)$ também integram categoria de gelatinas.

Os emulsificantes (36\%), fermentos químicos $(33 \%)$ e espessantes (24\%) foram encontrados com alta frequência em virtude das categorias analisadas no presente estudo. Os emulsificantes e espessantes são encontrados nas bebidas lácteas $(15 \%$ e $44 \%)$ e iogurtes $(9 \%$ e $71 \%)$ e os fermentos estão muito presentes na categoria dos biscoitos recheados (88\%), além dos bolos (100\%), porém, nesta categoria, são componentes intrínsecos da preparação segundo o Regulamento Especifico de utilização de Fermentos ${ }^{[12]}$.

A análise de cluster resultou em seis grupos, sendo que os três primeiros clusteres, apresentam categorias de produtos alimentícios e classes de aditivos diversas, com predominância de biscoito recheado e cereal matinal, respectivamente. $\mathrm{O}$ terceiro cluster engloba todos os produtos da categoria de gelatinas, mas é majoritariamente composto por bebidas à base de frutas. Já os três últimos clusteres foram compostos predominantemente por iogurtes e bolos e salgadinhos de milho, com menor diversidade de classes de aditivos que os demais grupos (Tabela 2). Os clusters foram criados desconsiderando-se as categorias de gelificante, melhorador de farinha e antiespumante cujas frequências foram inferiores a $2 \%$.

Tabela 2. Frequência de categorias e aditivos nas seis categorias de clusteres geradas nos 409 produtos destinados ao público infantil avaliados, Belo Horizonte-MG 2018.

\begin{tabular}{|c|c|c|c|c|c|c|}
\hline & $\begin{array}{c}1 \\
(n=121)\end{array}$ & $\begin{array}{c}2 \\
(n=80)\end{array}$ & $\begin{array}{c}3 \\
(n=110)\end{array}$ & $\begin{array}{c}4 \\
(n=33)\end{array}$ & $\begin{array}{c}5 \\
(n=28)\end{array}$ & $\begin{array}{c}6 \\
(n=35)\end{array}$ \\
\hline \multicolumn{7}{|l|}{ CATEGORIAS } \\
\hline Bebidas à base de frutas & $0,8 \%(1)$ & $15,0 \%(12)$ & $47,3 \%(52)$ & - & - & - \\
\hline Bebidas lácteas & $2,5 \%(3)$ & $33,8 \%(27)$ & $0,9 \%(1)$ & $3,0 \%(1)$ & - & - \\
\hline Biscoito recheado & $83,5 \%(101)$ & $2,5 \%(2)$ & - & - & - & - \\
\hline Bolo & $9,9 \%(12)$ & $3,8 \%(3)$ & - & - & $100 \%(28)$ & - \\
\hline Cereal matinal & - & $37,5 \%(30)$ & - & - & - & - \\
\hline Gelatina & - & - & $45,5 \%(50)$ & - & - & - \\
\hline Iogurte & - & $1,3 \%(1)$ & - & $97,0 \%(32)$ & - & - \\
\hline Salgadinho & $3,3 \%(4)$ & $6,3 \%(5)$ & $5,5 \%(6)$ & - & - & $100 \%(35)$ \\
\hline \multicolumn{7}{|l|}{ ADITIVOS } \\
\hline Aromatizantes & $96,7 \%(117)$ & $67,5 \%(26)$ & $99,1 \%(109)$ & $97,0 \%(32)$ & $100 \%(28)$ & $100 \%(35)$ \\
\hline Acidulantes & $19,8 \%(24)$ & $1,3 \%(1)$ & $4,5 \%(5)$ & $97 \%(32)$ & $100 \%(28)$ & $20,0 \%(7)$ \\
\hline Antioxidante & - & $15,0 \%(12)$ & $10,0 \%(11)$ & - & - & - \\
\hline Antiumectantes & $0,8 \%(1)$ & $7,5 \%(6)$ & $5,5 \%(6)$ & - & $35,7 \%(10)$ & $31,4 \%(11)$ \\
\hline Corantes & $57,9 \%(70)$ & $18,8 \%(15)$ & $68,2 \%(75)$ & $90,9 \%(30)$ & $14,3 \%(4)$ & $82,9 \%(29)$ \\
\hline Conservantes & $9,9 \%(12)$ & - & $4,5 \%(5)$ & $100 \%(33)$ & $100 \%(28)$ & - \\
\hline Edulcorantes & $0,8 \%(1)$ & - & $58,2 \%(64)$ & $3,0 \%(1)$ & - & - \\
\hline Emulsificantes & $99,2 \%(120)$ & $3,8 \%(3)$ & $5,5 \%(6)$ & $30,3 \%(10)$ & $92,9 \%(26)$ & - \\
\hline Espessantes & $2,5 \%(3)$ & $16,3 \%(13)$ & $37,3 \%(41)$ & $75,8 \%(25)$ & $42,9 \%(12)$ & - \\
\hline Estabilizante & $3,3 \%(4)$ & $45,0 \%(36)$ & $3,6 \%(4)$ & $51,5 \%(17)$ & - & - \\
\hline Regulador de acidez & $3,3 \%(4)$ & $1,3 \%(1)$ & $99,1 \%$ (109) & - & - & $20,0 \%(7)$ \\
\hline Umectantes & $21,5 \%(26)$ & - & - & - & $100 \%(28)$ & - \\
\hline Realçadores de sabor & $3,3 \%(4)$ & - & $5,5 \%(6)$ & - & - & $100 \%(35)$ \\
\hline
\end{tabular}




\section{DISCUSSÃO}

$\mathrm{Na}$ amostra estudada, foram encontrados 17 das 23 classes de aditivos com utilização respaldada pelo Codex ${ }^{[3]}$. O número de produtos que apresentavam aditivos foi 95,4\%. Sendo assim, são ínfimas as chances do consumidor conseguir adquirir um produto industrializado livre de aditivos alimentares, o que vai contra a indicação do Guia Alimentar para a População Brasileira de restringir tais alimentos do hábito alimentar ${ }^{[4]}$.

A classe dos aromatizantes $(88,0 \%)$ foi a mais encontrada, presente em todas as categorias analisadas. Os aromatizantes estiveram presentes em todos os seis clusteres gerados, juntamente com os corantes. Acredita-se que o uso trivial de aromatizantes esteja relacionado com melhoria sensorial dos produtos, como citado por Polônio e colaboradores[10]. Dessa forma, os produtos ultraprocessados agradam o paladar, principalmente da criança que ainda está em formação dos hábitos alimentares, tornando-o pertencente ao padrão alimentar das mesmas ${ }^{[6]}$.

A grande presença de aromatizantes nos produtos do presente estudo corrobora com estudo realizado com alunos $(n=160)$ do ensino médio de Alagoas no qual, por meio de questionário de consumo, verificou-se que existe consumo exacerbado dos alimentos com alta diversidade de aditivos, sendo mais consumido os aromatizantes[13]. Não foi possível identificar quais eram os aromatizantes empregados, uma vez que a RDC no 2 de 2007 permite que eles sejam declarados de forma agrupada, dificultando a separação destes em naturais e sintéticos ${ }^{[11]}$.

No presente estudo, os corantes foram a segunda classe de aditivos mais encontrada, $56 \%$ da amostra continha algum tipo, seja natural, idêntico ao natural ou sintético. É possível que combinações de vários corantes sejam utilizadas para alcançar a cor desejada, comprovando a hipótese existente da utilização dos aditivos alimentares como forma de apelo sensorial[14,15]. Polônio e colaboradores demonstraram, por meio de estudo transversal observacional, como é fácil para as crianças ultrapassarem a IDA de corantes artificiais, devido a sua prevalência em todas as categorias de alimentos destinadas a esse público[10].
Edulcorante é mais uma classes de aditivos que necessita de atenção frente ao apelo sensorial ofertado, uma vez que, tanto os naturais como os artificiais, apresentam associação com o consumo excessivo de alimentos, principalmente, por mascarar os sentidos de saciedade e saciação[16]. Estes foram encontrados em $16,4 \%$ da amostra do presente estudo, estando presente em três categorias especificas: gelatinas, bebidas à base de fruta e iogurtes. Em sua maioria, os produtos apresentaram combinações de substâncias, como acessulfame de potássio e aspartame. Acredita-se que o intuito da utilização dessas substâncias é reduzir os teores de açúcares e, consequentemente, peso dos produtos e o frete, porém, não existe nenhuma comprovação que justifique tal substituição.

Conservantes $(19 \%)$, antioxidantes $(6 \%)$ e realçador de sabor $(11 \%)$ apresentaram menor frequência na análise do presente estudo. Acredita-se que tal dado foi influenciado pelas categorias que compõe a amostra, isso porque algumas categorias são restritas a alimentos específicos, como por exemplo o conservante nitrito que é restrito a produtos cárneos ${ }^{[3]}$.

Resultados de estudos experimentais com animais demonstram que, em altas doses, os antioxidantes desencadearam problemas hepáticos e gastrointestinais ${ }^{[17]}$. Estudos do efeito do consumo de antioxidantes em quantidades contidas em produtos alimentícios, de forma crônica em humanos, são necessários, uma vez que foram encontrados em $33 \%$ dos cereais matinais e $20 \%$ das bebidas à base de fruta.

No presente estudo foram encontrados repetição e prevalência de aditivos na amostra como um todo e nas médias de aditivos por categoria. O resultado da análise de cluster corrobora os demais achados, uma vez que os cluster não englobaram apenas uma categoria de produto como era esperado, devido a grande variedade de aditivos alimentares empregados pela Indústria de Alimentos. Principalmente, pelo número limitado de estudos que analisam o consumo de vários aditivos alimentares de forma crônica. Uma vez que todos os trabalhos encontrados tratam de forma isolada de um único aditivo ou de uma única categoria. 
A heterogeneidade dos grupos, tanto ao analisar as categorias de alimentos como as classes de aditivos, encontrados nesta analise demonstra como os critérios para utilização de aditivos não seguem o padrão adequado, apesar do Codex ressaltar que devem ser empregados segundo suas funções e, apenas, na quantidade mínima necessária para que essa seja exercida de maneira eficaz ${ }^{[3]}$. Assim, acredita-se que sejam usados para gerar maior aceitação desses alimentos, por proporcionar texturas, cores e sabores ainda mais atraentes ${ }^{[1]}$.

Além disso, para justificar a preocupação com o consumo de aditivos alimentares, um estudo de coorte francês associou dieta baseada em alimentos ultraprocessados, mesmo com o controle de nutrientes críticos, e alto risco de mortalidade ${ }^{18]}$. Sendo considerado um dos possíveis efeitos prejudiciais de tais produtos alimentícios a utilização dos aditivos alimentares ${ }^{[18]}$. Tais achados devem ser levados em consideração para que haja maior direcionamento das políticas públicas a nível nacional e mundial, minimizando os impactos e, consequentemente, os custos com saúde [19]. Diante dos riscos associados ao consumo de aditivos, devese valorizar a alimentação baseada no proposto pelo Guia Alimentar da População Brasileira, respaldado pela classificação NOVA[2,4].

No presente estudo, não foi possível avaliar as quantidades de aditivos alimentares presentes nos produtos analisados, uma vez que o trabalho foi realizado através da declaração presente na lista de ingredientes e a legislação não exige que seja especificada tal informação. Embora as legislações vigentes indiquem quantidade máxima de aditivos a serem utilizadas baseadas no seu efeito individual, não há estudos que apontem malefícios sobre o uso vários de aditivos, em especial em longo prazo.

\section{CONCLUSÃO}

Conclui-se que os aditivos alimentares estão presentes na maioria absoluta dos produtos destinados ao público infantil. São encontradas com maior frequência as classes dos aromatizantes, corantes e emulsificantes, principalmente, nas gelatinas e nos bolos, o que corrobora com outros estudos. Esses dados evidenciam a necessidade de políticas públicas com o intuito de diminuir o consumo de alimentos ultraprocessados, resgatando assim hábitos alimentares saudáveis.

\section{REFERÊNCIAS}

[1] Monteiro CA, Moubarac JC, Cannon G, Ng SW, Popkin B. Ultra-processed products are becoming dominant in the global food system. Obes Rev. 2013;14(S2):21-8.

[2] Monteiro CA, Laura M. diet quality, and health using the NOVA classification system Prepared by. 2019.

[3] Codex Alimentarius, FAO (Organización de las Naciones Unidas para la Alimentación y la Agricultura), OMS (Organización Mundial de la Salud). Norma General del Codex para los Aditivos Alimentarios. 2018;507. Available from: http://www.fao.org/gsfaonline/docs/CXS_192s.pdf

[4] BRASIL. Guia alimentar para a população brasileira. Ministério da Saúde. 2014. 158 p.

[5] Rodrigues VM, Rayner M, Fernandes AC, De Oliveira RC, Da Costa Proença RP, Fiates GMR. Comparison of the nutritional content of products, with and without nutrient claims, targeted at children in Brazil. Br J Nutr. 2016;115(11):2047-56.

[6] Hinnig P de F, Bergamaschi DP. Itens alimentares no consumo alimentar de crianças de 7 a 10 anos. Rev Bras Epidemiol [nternet]. 2012;15(2):324-34. Available from: http://www.scielo.br/scielo.php?script=sci_arttext\&pid $=$ S1415-790X2012000200010\&lng $=$ pt\&tlng $=$ pt

[7] FERREIRA F de S. Aditivos Alimentares E Suas Reações Adversas No Consumo Infantil. Rev Da Univ Val Do Rio Verde [Internet]. 2015;397-407. Available from: http://periodicos.unincor.br/index.php/revistaunincor/ article/view/1845

[8] Ferreira JSG, Silva Y Da, Moraes OMG de, Tancredi RP. Marketing de alimentos industrializados destinados ao público infantil na perspectiva da rotulagem. Vigilância Sanitária em Debate. 2015;0(0):75-84.

[9] Lima M, Ares G, Deliza R. How do front of pack nutrition labels affect healthfulness perception of foods targeted at children? Insights from Brazilian children and parents. Food Qual Prefer. 2018;64:111-9. 
[10] Polônio MLT, Peres F. Consumo De Corantes Artificiais Por Pré-Escolares De Um Município Da Baixada Fluminense, Rj Consumo. 2012;4(1):2748-57.

[11] MAPA. Resolução RDC nำ 5, de 15 de janeiro de 2007. Ministério da Agric Pecuária e Abast. 2007;2007:ANEXO.

[12] King S, King H. Brasil. Resolução - CNNPA no 38 de 1977. 1977;7. 1977;(1):541-59.

[13] Albuquerque MV, dos Santos SA, Cerqueira N, da Silva JA. Educação Alimentar: Uma Proposta de Redução do Consumo de Aditivos Alimentares. Soc Bras Química [Internet]. 2012;34(02):51-7. Available from: http://qnint.sbq.org.br/qni/visualizar'Tema.php?idTema $=59$

[14] Bissacotti AP, Angst CA, Saccol AL de F. Implicações dos aditivos químicos na saúde do consumidor. Ciências da Saúde, St Maria [Internet]. 2015;16(1):43-59. Available from:

https://www.periodicos.unifra.br/index.php/disciplinaru $\mathrm{mS} /$ article/viewFile/1108/1050

[15] Instituto Brasileiro de Geografia e Estatística IBGE. Pesquisa Nacional de Saúde do Escolar: 2015 / IBGE, Coordenação de População e Indicadores Sociais. 2016. $132 \mathrm{p}$.
[16] Effects of long-term administration of aspartame on biochemical indices lipid profile and redox status of cellular system of male rats.

[17] Sasaki YF, Kawaguchi S, Kamaya A, Ohshita M, Kabasawa K, Iwama K, et al. The comet assay with 8 mouse organs: Results with 39 currently used food additives. Mutat Res - Genet Toxicol Environ Mutagen. 2002;519(1-2):103-19.

[18] Schnabel L, Kesse-Guyot E, Allès B, Touvier M, Srour B, Hercberg S, et al. Association between Ultraprocessed Food Consumption and Risk of Mortality among Middle-aged Adults in France. JAMA Intern Med. 2019;179(4):490-8.

[19] Polônio MLT, Peres F. Consumo de aditivos alimentares e efeitos à saúde: desafios para a saúde pública brasileira. Cad Saude Publica [Internet]. 2009;25(8):165366. Available from: http://www.scielo.br/scielo.php?script=sci_arttext\&pid $=$ S0102-311X2009000800002\&lng $=$ pt\&tlng $=$ pt 